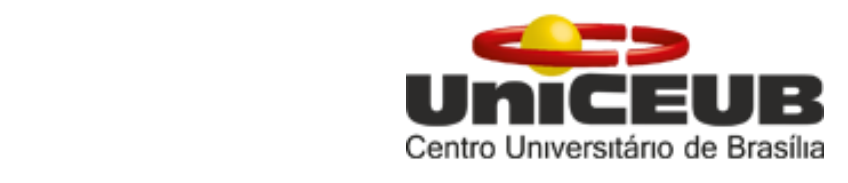

CENTRO UNIVERSITÁRIO DE BRASÍLIA - UnICEUB PROGRAMA DE INICIAÇÃO CIENTíFICA

Ana Carolina Mota de Faria

ESTUDO RETROSPECTIVO DA ROTINA CLÍNICA

BRASÍLIA

2018 


\section{$\longrightarrow$ \\ Unic: $=\mathbf{B}$ \\ Centro Unıversıtárıo de Brasílıa}

\section{Ana Carolina Mota de Faria}

\section{ESTUDO RETROSPECTIVO DA ROTINA CLÍNICA}

Relatório final de pesquisa de Iniciação Científica apresentado à Assessoria de Pós-Graduação e Pesquisa.

Orientação: Carlos Alberto da Cruz Júnior

\section{BRASÍLIA}


RESUMO

\title{
ESTUDO RETROSPECTIVO DA ROTINA CLÍNICA
}

\author{
Ana Carolina Mota de Faria - UniCEUB, PIC VETERINARI \\ ana.faria@sempreceub.com
}

Daniela Oliveira Rodrigues - UniCEUB, pesquisadora colaboradora

daniela.rodrigues@sempreceub.com

\section{Bruno Alvarenga dos Santos - UniCEUB, professor colaborador brunoalvarenga@gmail.com}

\section{Carlos Alberto da Cruz Júnior - UniCEUB, professor orientador carlos.junior@uniceub.br}

Com o aumento da população mundial de animais domésticos, manifesta-se uma crescente necessidade de atendimentos veterinários específicos que contribuam para uma melhor qualidade de vida dos animais. Os estudos de casuísticas em hospitais veterinários, com avaliação das afecções mais frequentes da rotina, tornam-se fundamentais para os processos de planejamento, prevenção e do tratamento adequado das espécies. Diante do exposto, realizou-se durante 4 meses consecutivos um estudo de coorte retrospectiva nos prontuários do Hospital Veterinari. A classificação das afecções clínicas foram baseadas nas informações contida nos prontuários e classificadas por sistemas corporais. Os pacientes que apresentaram mais de uma enfermidade, foram incluídos em dois ou mais sistemas. Foram avaliados 872 atendimentos que incluíram um total de 354 animais. A frequência de atendimento das espécies foi superior em caninos (86\%) quando comparado aos felinos (14\%), corroborando para os dados da literatura, na qual a população de cães é mundialmente maior que a de gatos. Durante o levantamento dos dados, observou uma maior frequência de animais do sexo masculino entre os caninos (53\%) e os felinos (56\%). Os principais atendimentos consistiram na imunização dos filhotes $(\mathrm{n}=265,28 \%)$, enquanto os diagnósticos mais recorrentes foram do sistema tegumentar $(n=133,14 \%)$, digestório $(n=121,13 \%)$, ortopedia $(n=94,10 \%)$, reprodutivo ( $n=51,5.3 \%)$, oftalmológico $(n=47,5 \%)$, oncológico $(n=42,4.4 \%)$, neurológico e toxicológico $(n=41,4.3 \%)$, urinário $(n=26,4.3 \%)$, odontológico $(n=25$, $2.6 \%)$, respiratóriob $(n=24,2.5 \%)$, infectocontagioso $(n=19,2 \%)$, endócrino $(n=15$, $2 \%)$, cardíaco $(n=5,0.5 \%)$ e outros $(n=45,4.7 \%)$. Os resultados evidenciaram uma maior procura por procedimentos de vacinação dos filhotes, essencial para a prevenção de doenças. Por outro lado, dentre os atendimentos mais recorrentes, observou-se um elevado número de afecções dermatológicas, que na maioria dos casos estava relacionado com reações alérgicas causados por fungos ou bactérias. Destacaram-se também nos demais atendimentos: casos periódicos de gastroenterite no aparelho digestório, fraturas e traumas musculares decorrentes de brigas e atropelamentos nos casos ortopédicos, partos distorcidos e piometra evolvendo o sistema reprodutor, enquanto nas enfermidades infectocontagiosas destacaram-se os casos de verminoses, cinomose e parvovirose. Com base no estudo, foi possível identificar os casos que mais ocorrem na rotina da clínica, em que a maioria das enfermidades apresentadas poderiam ser minimizadas ou evitadas por meio de medidas profiláticas na saúde animal.

Palavras-Chave: Doenças. Veterinária. Pacientes. 


\section{SUMÁRIO}

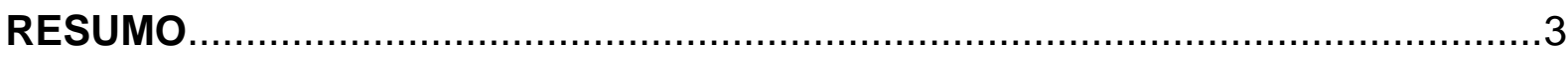

1. INTRODUÇÃO .....................................................................................

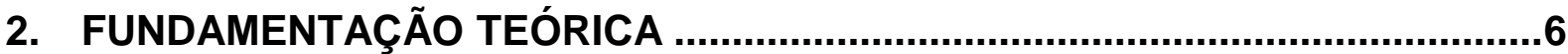

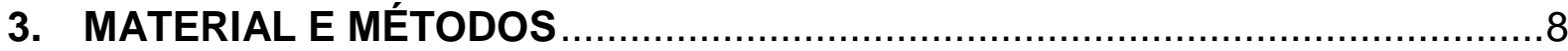

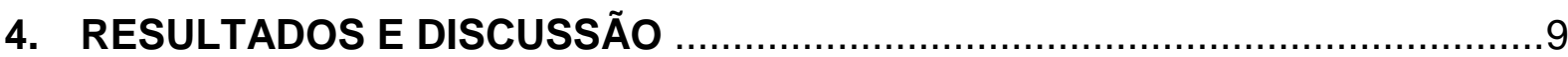

Figura 1. Casuística das primeiras espécies atendidas no Hospital Veterinari ...........9

Tabela 1. Padrão Racial de cães e gatos no hospital veterinari de Brasília............10

Figura 2. Idade dos animais atendidos no Hospital Veterinari entre o período de

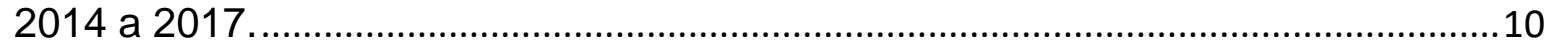

Figura 3. Relação entre espécies e sexo atendidos no Hospital Veterinari.............11

Tabela 2. Casuística das afecções clínicas entre o período de 2014 a 2017 .........12

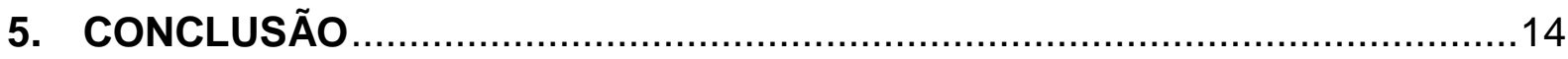

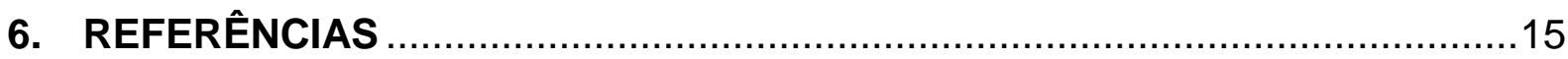




\section{INTRODUÇÃO}

Nos últimos anos, a sociedade moderna intensificou o vínculo entre os humanos e os animais, no que as espécies domésticas passaram a ser consideradas um membro familiar, principalmente em famílias que possuem idosos e crianças. Essa relação proporciona interações emocionais, psicológicas e físicas que envolve pessoas, animais e o meio ambiente (PIRES, 2009).

Com o aumento da população mundial de cães e gatos, manifestou-se uma crescente necessidade de atendimentos veterinários específicos que contribuem para uma melhor qualidade de vida dos animais, visto que a interação entre humano-animal vem ocupando papel de destaque na população brasileira (XAVIER, 2012).

O estudo epidemiológico em clínicas e hospitais veterinários constituem uma ferramenta importante para os processos de planejamento, prevenção e controle das afecções. Além disso, é fundamental o conhecimento do manejo e o controle sanitário eficiente dos animais na rotina veterinária, visto que estes podem atuar como disseminadores ou reservatórios de doenças (BEN 2014; BORTULUCCI et al., 2014).

Dessa forma, compreender o comportamento das doenças dentro da saúde animal e ambiental são essenciais para impedir a disseminação entres os animais, e por consequência, nos seres humanos (FIGUEIREDO et. al., 2001). Entretanto, poucos estudos relatam as casuísticas indicativas das moléstias relacionados à clínica de pequenos animais, abordando fatores como o perfil dos animais atendidos, as afecções clínicas e o tipo de tratamento ao qual o animal foi submetido (CRUZ-PINTO et al., 2015). Por outro lado, o diagnóstico precoce, aliado ao tratamento eficaz podem minimizar essas problemáticas (ALVES \& SANTOS et al 2017).

Em vista do aumento de atendimentos na clínica de pequenos animais e da devida importância, este trabalho teve como objetivo traçar um perfil epidemiológico das afecções mais recorrentes da rotina clínica veterinária, para atuar nas prevenções e no controle das enfermidades, além de reportar informações para as tomadas de decisões gerenciais. 


\section{FUNDAMENTAÇÃO TEÓRICA}

A epidemiologia é a ciência que avalia todos os fatores envolvidos que desencadeiam uma doença. Assim, a pesquisa científica possui uma grande importância no reconhecimento dos problemas, em que é possível definir a sua casualidade, determinar a natureza, e consequentemente, esclarecer um tratamento e desenvolver medidas de prevenção (OILIVEIRA et al 2010).

$\mathrm{Na}$ medicina veterinária, o campo de maior expansão na ciência está relacionado a clínica de pequenos animais. Para Fischer \& Petrucci (2005), o médico veterinário deve conhecer o comportamento biológico dos animais, além de procurar maneiras de preveni-las, não somente estabelecendo o diagnóstico das doenças na rotina da clínica veterinária.

Para BEN (2014), é fundamental uma anamnese bem elaborada que forneça bases sólidas para um diagnóstico preciso, além da utilização de exames laboratoriais pertinentes. Sendo que estes, portanto, mostram-se como ferramentas de auxílio que permitem o melhor monitoramento e o indicativo da saúde dos animais.

Os animais de companhia podem apresentar-se como potenciais transmissores de doenças aos tutores, e o diagnóstico precoce pode minimizar a possibilidade dos riscos. Assim, os conhecimentos da distribuição das principais enfermidades da rotina clínica podem auxiliar os profissionais na tomada de decisão, além de traçar um perfil epidemiológico da população atendida (SANTOS, 2006).

No estudo de Chamas et al (2010), identificou-se que $10 \%$ dos atendimentos clínicos representavam quadro de doenças infectocontagiosas, apresentando-se como grande relevância para a saúde pública. Enquanto a pesquisa de Xavier (2012) demonstrou uma maior casuística de atendimentos relacionados às especialidades dermatológicas (19\%) e digestórias (18\%), sendo que as infecciosas ocuparam 15\% da casuística. Chamas et al (2011) também ressaltou a importância na avaliação periódica constante de cães machos idosos, buscando o diagnóstico e o tratamento precoce das afecções de insuficiência cardíaca, onde estes são mais frequentemente acometidos.

Em cadelas, as afecções em glândulas mamárias são a principal causa de tumores, enquanto essas ocorrências em machos são incomuns (SANTOS, 2010). Para Pereira (2010), as lesões ortopédicas estão relacionadas principalmente no joelho, devido a quedas, sobrepeso ou torções em pisos escorregadios. 
Diante disso, o estudo das enfermidades na rotina da clínica de pequenos animais constitui um fator determinante para a prevenção e controle das doenças, através do melhor conhecimento das situações, dos fatores determinantes e das melhores formas de cura e reabilitação dos pacientes. Além disso, os profissionais de saúde procuram sempre por dispositivos e subsídios que enriqueçam sua prática e potencializem a experiência da clínica veterinária, buscando análises críticas e aplicação das informações científicas (ALVES \& SANTOS, 2017; FARACO, 2008). 


\section{MATERIAL E MÉTODOS}

Realizou-se um estudo retrospectivo durante 4 meses consecutivos de cães e gatos atendidos no Hospital Veterinari, localizado em Brasília - DF. Foram avaliadas 872 fichas clínicas que incluíram um total de 354 animais. A classificação das afecções clinicas foram baseadas nas informações contida nos prontuários e classificadas por sistemas corporais, que incluíram atendimentos de imunização, sistema tegumentar, digestório, ortopédico, reprodutivo, oftalmológico, oncológico, neurológico e toxicológico, urinário, odontológico, respiratório, infectocontagioso, endocrinológico e outros. Os pacientes que apresentaram mais de uma enfermidade, foram incluídos em dois ou mais sistemas.

A ficha clínica apresentava informações com relação aos dados dos proprietários e dos pacientes atendidos. Os dados coletados durante o estudo foram: espécie, padrão racial, idade, sexo, afecção clínica, afecção sugestiva, prescrição. Além disso, as idades dos animais foram classificadas e agrupadas em: $1-3$ anos (Animais jovens), 4-10 anos (Animais adultos) e acima de 11 anos os animais idosos. Os dados coletados foram tabelados e submetidos a análise estatística.

Para avaliar os resultados da pesquisa, foi empregado o método de descrição analítica, por meio da tabulação de dados no programa Microsoft Office Excel 2010, onde o cálculo foi realizado por meio dos valores expressos em porcentagem. 


\section{RESULTADOS E DISCUSSÃO}

Durante o estudo da rotina clínica, observou-se que a frequência de atendimento em cães $(n=751,84,1 \%)$ foi superior à de gatos $(n=121,13,5 \%)$, indo de encontro com pesquisas feitas pelo IBGE, no qual indicava uma maior população canina entre as famílias brasileiras quando comparado aos felinos. As aves representaram $1,7 \%(n=15)$ atendimentos e outros animais, como roedores e espécies silvestres $0,7 \%(n=6)$ da casuística do hospital (Figura 1).

Figura 1. Casuística das primeiras espécies atendidas no Hospital Veterinari

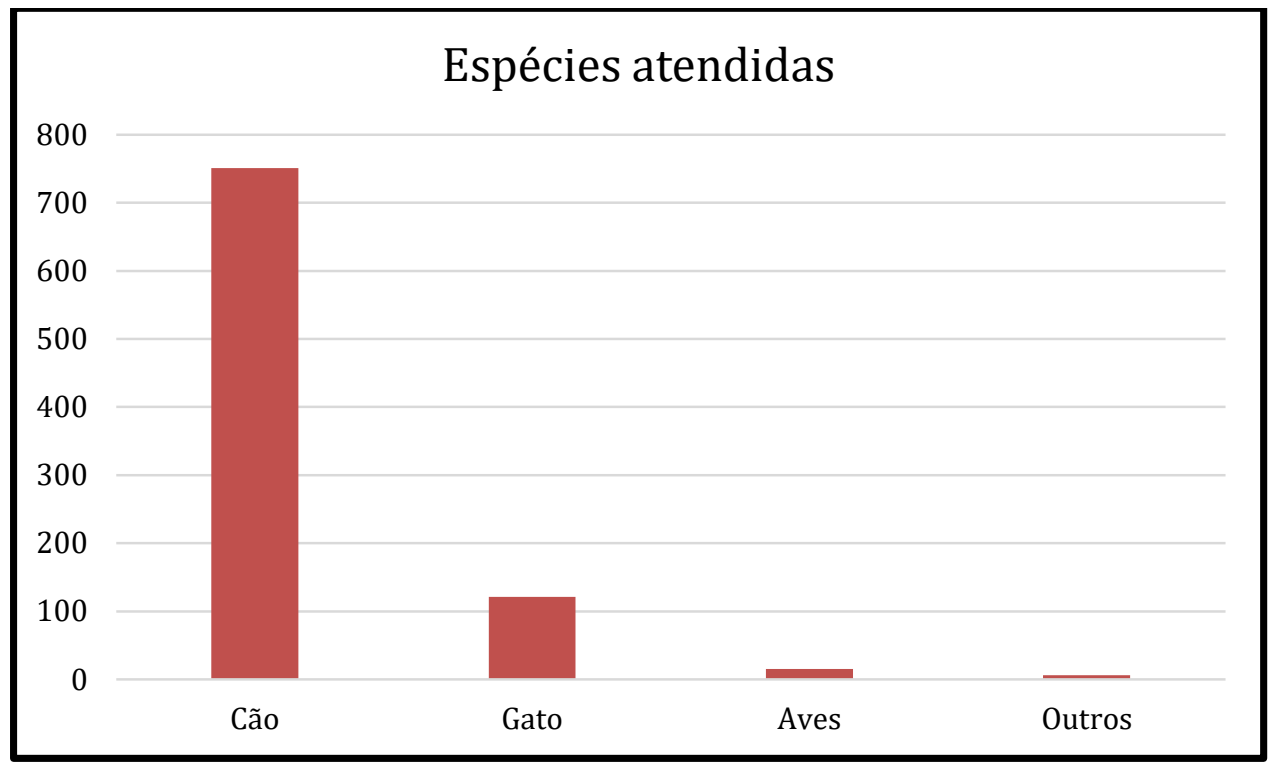

Fonte de dados: Hospital Veterinari, 2014

Enquanto as raças dos animais, foi observado uma maior ocorrência de cães e gatos SRD ( $n=190,25,30 \%$ e $n=68,56,20 \%$, respectivamente) como exibido na tabela 1. Os resultados também foram observados por diversos trabalhos que evidenciam um maior atendimento de animais SRD (ALVES \& SANTOS, 2017). 
Tabela 1. Padrão Racial de cães e gatos no hospital veterinari de Brasília.

\begin{tabular}{|llllll|}
\hline \multicolumn{7}{|c|}{ Padrão Racial } \\
\hline Cães & $\mathbf{n}^{\mathbf{1}}$ & $\mathbf{\%}$ & Gatos & $\mathbf{n}^{\mathbf{2}}$ & $\%$ \\
\hline SRD & 190 & 25,30 & SRD & 68 & 56,20 \\
Shih -Tzu & 107 & 14,25 & Persa & 43 & 35,54 \\
Maltês & 80 & 10,65 & Siamês & 8 & 6,61 \\
Yorkshire & 70 & 9,32 & Himalaio & 2 & 1,65 \\
Labrador & 52 & 6,92 & Outros & 0 & 0,00 \\
Outros & 252 & 33,56 & - & - & - \\
\hline Total & $\mathbf{7 5 1}$ & 100,00 & Total & $\mathbf{1 2 1}$ & 100,00 \\
\hline $\mathrm{n}^{1}$ quantidade de cães $\mathrm{n}^{\mathbf{2}}$ quantidade de gatos \\
\hline
\end{tabular}

Fonte de dados: Hospital Veterinari, 2014.

Enquanto a faixa etária dos animais atendidos variou entre 6 meses a 20 anos, obtendo uma maior prevalência de atendimentos em animais jovens $(<1-3$ anos, $n=$ $49,8 \%$ ), seguido de adultos (4-10 anos, $36,7 \%$ ), e os animais idosos (acima de 11 anos, 13,5\%) como apresentado na Figura 2.

Figura 2. Idade dos animais atendidos no Hospital Veterinari entre o período de 2014 a 2017.

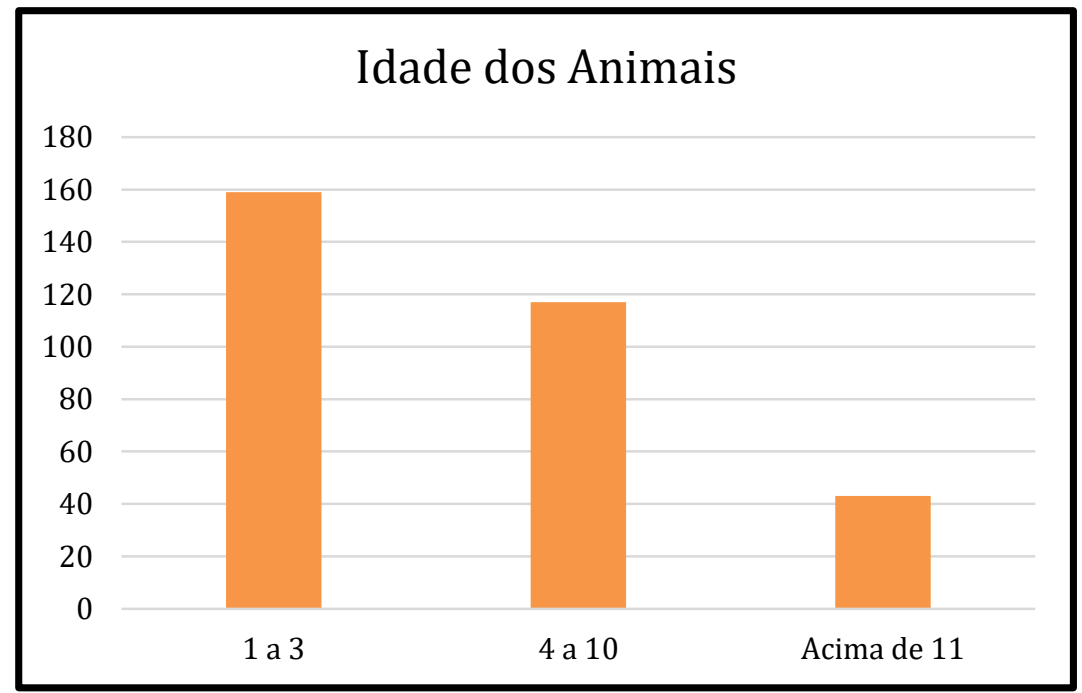

Fonte de dados: Hospital Veterinari, 2014

Em relação ao sexo (Figura 3), na espécie canina a maior frequência de atendimento foi em machos $(53,8 \%)$, o que também ocorreu na espécie felina $(53,2 \%)$. 
Figura 3. Relação entre espécies e sexo atendidos no Hospital Veterinari.

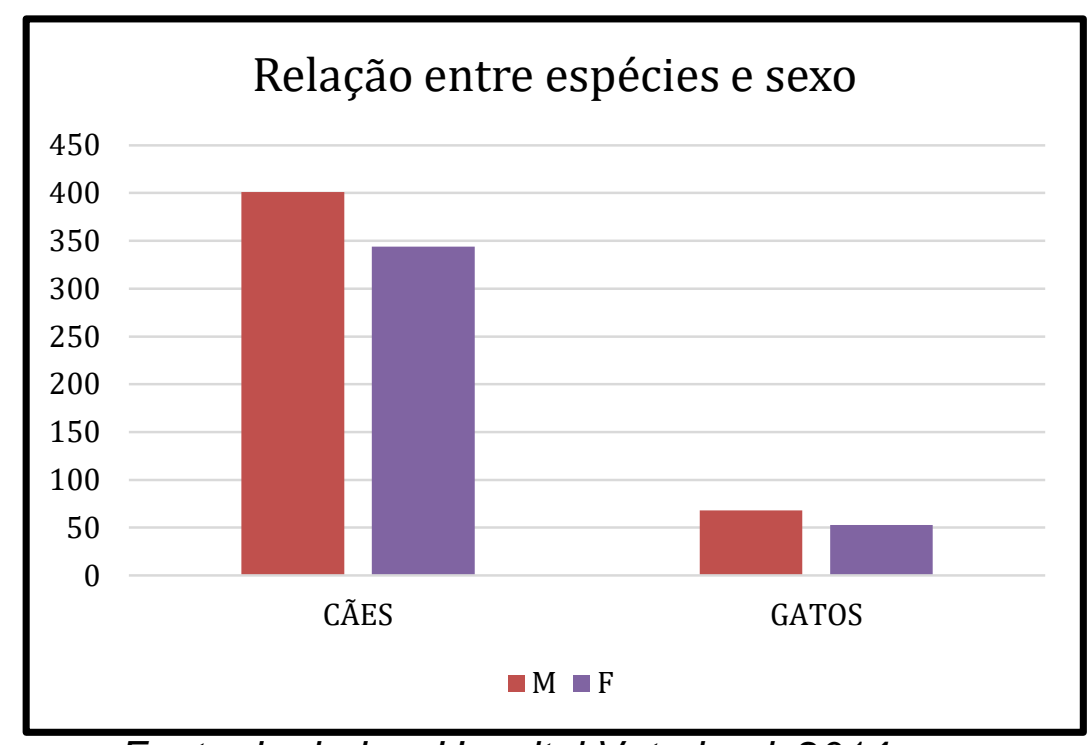

Fonte de dados: Hospital Veterinari, 2014

Os principais atendimentos consistiram na imunização dos filhotes $(n=265$, $28 \%$ ), enquanto os diagnósticos mais recorrentes foram do sistema tegumentar ( $\mathrm{n}=$ $133,14 \%)$, digestório $(n=121,13 \%)$, ortopedia $(n=94,10 \%)$, reprodutivo $(n=51,5.3 \%)$, oftalmológico ( $n=47,5 \%)$, oncológico $(n=42,4.4 \%)$, neurológico e toxicológico $(n=41$, $4.3 \%)$, urinário $(n=26,4.3 \%)$, odontológico $(n=25,2.6 \%)$, respiratório $(n=24,2.5 \%)$, infectocontagioso $(n=19,2 \%)$, endócrino $(n=15,2 \%)$, cardíaco $(n=5,0.5 \%)$ e outros $(n=45,4.7 \%)$ como exibido na tabela 2 . 
Tabela 2. Casuística das afecções clínicas entre o período de 2014 a 2017.

\begin{tabular}{|lrr|}
\hline \multicolumn{1}{c}{ Afecção Clínica } & \multicolumn{3}{c}{$\%$} \\
\hline Imunização & 265 & 27,9 \\
Tegumentar & 133 & 13,95 \\
Digestório & 121 & 12,69 \\
Ortopedia & 94 & 9,86 \\
Reprodutivo & 51 & 5,35 \\
Oftamológico & 47 & 4,93 \\
Oncológico & 42 & 4,41 \\
Neuro e Toxicológico & 41 & 4,3 \\
Urinário & 26 & 2,73 \\
Odontologia & 25 & 2,62 \\
Respiratório & 24 & 2,52 \\
Infecto-Contagiosa & 19 & 1,99 \\
Endocrino & 15 & 1,57 \\
Cardíaco & 5 & 0,52 \\
Outros & 45 & 4,72 \\
\hline Total & 953 & $\mathbf{1 0 0}$ \\
\hline 1 número de animais & & \\
\hline
\end{tabular}

Fonte de dados: Hospital Veterinari, 2014

Os resultados evidenciaram uma maior procura por procedimentos de vacinação dos filhotes (Tabela 2), essencial para a prevenção de doenças. Por outro lado, dentre os atendimentos mais recorrentes, observou-se um elevado número de afecções dermatológicas, em que na maioria dos casos, esteve relacionado a dermatites alérgicas, sarnas, otites, incluindo também as enfermidades parasitárias, colaborando com os estudos de Rosa et al (2011) e Xavier (2012).

Destacaram-se também nos demais atendimentos casos periódicos de gastroenterite alimentar no aparelho digestório, enquanto os mesmos resultados foram observados no estudo de Xavier (2012). Os atendimentos ortopédicos abrangeram casos de feridas, fraturas, luxações e claudicações, e que na maioria das vezes, estar relacionado a brigas e atropelamentos (BEN et al 2014). 
Dentre o sistema reprodutivo, $20 \%$ foram em machos e $80 \%$ em fêmeas, destacando-se a ovário-salpingo-histerectomia (60\%) como procedimento mais realizado. Além de casos de piometra e o parto distorcido, em que pode ser provocado por fatores maternos, fetais ou devido a contrações uterinas insuficientes, colaborando com a pesquisa de Silveira et al (2007).

Observou-se também uma maior ocorrência associada ao aparelho reprodutor $(n=41,30 \%)$. Nas cirurgias oncológicas, destacaram-se os procedimentos de mastectomias (53\%) sendo $81 \%$ em cadelas e $18 \%$ em gatas, indo de encontro ao observado por Portilho et al (2015).

Em relação as afeções urinárias, observou quadros de cistite e insuficiência renal. Os casos neurológicos estavam relacionados a doenças infecciosas, convulções e toxicológicos associados a casos de ingestão de medicamentos, produtos de limpeza ou por envenenamento. As afecções oftalmológicas estavam relacionadas a ocorrência de ulceração corneana, cataratas, e prolapsos da $3^{\circ}$ pálpebra, onde Slatter (2005) explica que são casos comuns e importantes para a rotina da clínica de pequenos animais, na qual exige tratamento correto para garantir resultados satisfatórios.

Os casos endócrinos estiveram relacionados a quadros de diabetes melitus, enquanto as afecções do sistema cardiológico mais comum eram sopros cardíacos e miocardiopatias. As enfermidades do trato respiratório foram relacionadas a tosse e casos de traqueobronquite, indo de encontro ao trabalho de Xavier (2012). Enquanto as afecções do sistema odontológico foram relacionadas a doenças periodontais e tratamento e restauração de canais.

Ressalta-se que os estudos epidemiológicos da rotina clínica de pequenos animais são importantes, principalmente para que seja possível traçar o perfil dos pacientes atendidos, para fornecer a adoção de medidas terapêuticas e profiláticas a esses pacientes. 


\section{CONCLUSÃO}

Com base no estudo, foi possível traçar o perfil de pacientes que frequentaram o Hospital, bem como identificar os casos que mais ocorrem na rotina da clínica, em que a maioria das enfermidades apresentadas poderiam ser minimizadas ou evitadas por meio de medidas profiláticas na saúde animal.

Dessa forma, sugere-se um fortalecimento contínuo do segmento de prevenção junto aos clientes por meio das informações dos prontuários que possibilitem uma melhoria continuada de processos relacionados a gestão da área cirúrgica veterinária. 


\section{REFERÊNCIAS}

ALVES, G.M; SANTOS, T.R. Estudo retrospectivo dos casos clínicos atendidos no serviço de clínica médica de pequenos animais do Centro Veterinário do UNIPAM. Revista Perquirere. Patos de Minas, n.14, v. 2, p. 1-11, maio/ago. 2017.

BEN, A.L et al. Casuística retrospectiva dos diagnósticos clínicos e solicitações de exams laboratoriais na rotina do HV-UEM, durante o período de 2011 e 2012. Revista de Ciência Veterinária e Saúde Pública, Maringá, v. 1, supl. 1, p. 059, 2014.

BORTULUCCI, D. E.; IANEGITZ, A. P.; BEN, A. L.; SANTANA, J. L. C.; WOSIACKI, S. R.; MUNHOZ, P. M. "Levantamento retrospectivo dos diagnósticos clínicos e procedimentos cirúrgicos relativos aos animais de pequeno porte no HV-UEM, período de 2011 e 2012". Revista de Ciência Veterinária e Saúde Pública, v. 1, supl. 1, p. 070, 2014.

CHAMAS, P.P.C.; SALDANHA, I.R.R.; COSTA, R.L.O. Prevalência da Doença Degenerativa Valvular Crônica Mitral em Cães. J Health Sci Inst, São Paulo, v. 29, p. 214-217, 2011.

CRUZ-PINTO, C. E.; STOPIGLIA, A. J.; MATERA, J. M.; ARNONI, F. I. Análise da casuística das afecções cirúrgicas observadas na Clínica Cirúrgica de Pequenos Animais da FMVZ-USP no período de 1988 a 2007. Brazilian Journal of Veterinary Research and Animal Science, v. 52, n. 1, p. 41-47, 13 abr. 2015.

FARACO, C. B. Interação humano-animal. Ciência veterinária nos trópicos. Editora Aurea Wischral, Recife, v. 11, ํo supl. 1, p. 31-35, jan/abr 2008.

FIGUEIREDO, C. M.; MOURÃO, A. C.; OlIVEIRA, M. A. A.; ALVES, W. R.; OOTEMAN, M. C.; CHAMONE, C. B.; KOURY, M. C. Leptospirose humana no município de Belo Horizonte, Minas Gerais, Brasil: uma abordagem geográfica. Revista da Sociedade Brasileira de Medicina Tropical, v. 34, n. 04, p. 331- 338, 2001.

FISHER, C.D.B; PETRUCCI, C.G.O. Estudo retrospectivo de casos clínicos atendidos na disciplina de Clínica Veterinária II da Faculdade de Veterinária da Universidade Luterana do Brasil (ULBRA), no período de agosto de 1999 a dezembro de 2004. Revista Veterinária em foco, Canoas, v.2, n.2, nov/abr, 2005.

IBGE - Instituto Brasileiro de Geografia e Estatísticas. População de animais de estimação no brasil. 2013.2 Disponível em: $<$ http://www.agricultura.gov.br/assuntos/camaras-setoriais tematicas/documentos/camaras-tematicas/insumos-agropecuarios/anos anteriores/ibge-populacao-de-animais-de-estimacao-no-brasil-2013-abinpet-79.pdf.> Acesso em: 15/03/2018.

OLIVEIRA, C.C.; OLIVEIRA, C.M.M.; HOLANDA, L.C.; BARBOSA, M.T.; ALBUQUERQUE, M.S.; MIRANDA, P.K.S.; SILVERIO, L.M.G.S.; FILHO, A.T.; 
FREITAS, A.A. Frequência Epidemiológica das doenças infecto-contagiosas em cães atendidos no Hospital Veterinário da UFRPE. Recife, 2010.

PEREIRA, D.S.S. Técnica de Reparação da Instabilidade da Articulação do Joelho em Caninos por Ruptura do Ligamento Cruzado Cranial. 2010. 40f. Dissertação de Tese (Monografia) - Departamento de Medicina Veterinária, Faculdades Integradas UPIS, Brasília- DF.

PIRES, A.M.C.L. Importância dos animais na socialização e no aprendizado de alunos do ensino fundamental. Em extensão, v.8, n.2, p. 158-161, 2009.

PORTILHO, C.A; ARREGUY, A.; DOS SANTOS, A.L.A. Estudo retrospectivo da casuística de cadelas e gatas com parto distócico atendidos no hospital veterinário univiçosa entre 2010 a 2014. ANAIS SIMPAC, v. 7, n. 1, 2017.

SANTOS, A.G. Perfil epidemiológico da população canina assistida pelo serviço de pronto atendimento do centro de controle de zoonoses Paulo Dacorso Filho, Dissertação de Tese (Mestrado). Rio de Janeiro, Brasil, Universidade Federal Rural do Rio de Janeiro, 2006. 64p.

SANTOS, W.P.P.; MONTENEGRO, G.E.S.; NOVACK, N.; FILHO, R.S.S.; JUNIOR, A.S.C.; MARANHÃO, F.E.C.B. Freqüência de Mastectomias realizadas em cadelas e gatas na rotina do Hospital Veterinário do Departamento de Medicina Veterinária da Universidade Rural de Pernambuco. Recife, 2010.

SLATTER, D. Fundamentos de Oftalmologia Veterinária. 3. ed. São Paulo: Rocca, 2005. 686p.

SILVEIRA, D.S.; BASSI, P.B.; OTERO, L.B.; SILVEIRA, L.W.; SOARES, N.N.; MENDES, T.C. Piometra em Caninos e Felinos: Perfil Leucocitário, Prevalência nas Espécies e Sinais Clínicos. 2007.

XAVIER, D.G. Casuística clínica e cirúrgica de uma clínica veterinária na cidade de camaquã/RS, durante o periodo de 2008 a 2011. 2012. Dissertação de Tese (Monografia). Universidade Rural do Semi-árido - UFERSA. 19p. 2012. 